\title{
In situ SECM detection of metal dissolution during zinc corrosion by means of mercury sphere-cap microelectrode tips
}

\author{
Ricardo M. Souto, Yaiza González-García, Dario Battistel, Salvatore Daniele
}

\begin{abstract}
This work presents a novel SECM-based in situ corrosion probing methodology that is capable of monitoring the release of zinc species in corrosion processes. It is based on the use of Hg-coated Pt-microelectrodes as SECM tips, which offer a wider negative potential range than bare platinum or other noble metals tips. This allows for the reduction of zinc ions at the tip to be investigated with low interference from hydrogen evolution and oxygen reduction from aqueous solutions. The processes involved in the corrosion of zinc during its immersion in chloride-containing solutions were successfully monitored by scanning the SECM-tip, set at an adequate potential, across the sample either in one direction or in the $X-Y$ plane parallel to its surface. In this way, it was possible to detect the anodic and cathodic sites where the dissolution of zinc and the reduction of oxygen occurred, respectively. Additionally, cyclic voltammetry $(\mathrm{CV})$ or constant potential measurements were used to monitor the release of zinc species collected at the tip during an SECM scan.
\end{abstract}




\section{Introduction}

Surface modification of metallic materials with polymer coatings constitutes an efficient protection procedure against corrosion as they introduce a physical barrier layer that separate the metal from the corrosive environment [1-4]. But these organic layers never provide an absolute separation between the underlying substrate and the electrolyte. In fact, the permeation of water and oxygen through the polymer matrix in defect-free coated metals immediately after exposure to aqueous electrolytes is well documented [5], whereas the development of ionic pathways through the polymer is regarded to occur prior to the transport to the metal-polymer interface of other aggressive species such as the chloride ions. That is, the organic film should have already become defective before the onset of corrosion processes at the buried metal substrate. But these ideas have been greatly shaken by recent reports that a specific effect of chloride ions towards swelling of the coating and even early blister initiation in a variety of metal-polymer systems could be observed by SECM operating in the feedback mode [6-9]. In contrast, blistering could not be observed in chloride-free solutions (namely sulphate, nitrate or borate solutions have been explored so far). The direct consequence of those observations is that chloride must enhance degradation of the coating at a very early stage, certainly at significantly shorter times that coating failure could be observed by either conventional electrochemical techniques or visual observation $[10,11]$.

The lack of confirmation of the reported effects from the use of other techniques, as well as the concern raised by the addition of redox mediators for SECM imaging, have imposed a certain caution as for the general acceptance of those observations. Conventional operation of the SECM in the feedback mode requires the measurement of a faradaic current at the sensing probe (i.e., the SECM tip) [12,13], and topographic changes in an insulating surface such as the defect-free coated metal originate from local hindrance in the diffusion-limited transport of the redox mediator as the tip is moved in close proximity to the surface. Yet, the absence of swelling effects in chloride-free electrolytes of similar ionic strength [10], as well as the recent report that the same effect has been imaged by SECM without the addition of an external redox mediator by using the naturally dissolved oxygen in the electrolyte [14], were strong evidences supporting the reported features.

To gain further confirmation on the existence of an early specific effect of chloride ions in defect-free coated metals, the use of a recently developed microelectrochemical technique, the alternating-current scanning electrochemical microscopy (AC-SECM) [15-20], was considered. This technique does not require redox reactions to happen for local imaging of 
surfaces, but it is based on highly sensitive determinations of the frequency-dependence of the local resistance at the tip, which is found to greatly depend on both the tip-to-sample separation and the electrochemical reactivity of the scanned substrate [21,22]. Experiments were conducted on coil-coated architectural cladding which consists of galvanised mild steel coated with a polyester resin. The galvanising coating consisted of galfan ( $95 \% \mathrm{Zn}+5 \% \mathrm{Al}$ ).

\section{2.- Experimental}

This work was carried out on a coil-coated steel (CCS) system consisting in a $0.4 \mathrm{~mm}$ mild steel foil coated with a $20 \mu \mathrm{m}$ galfan $(Z n-5 A /)$ coating, and this in turn being covered with 25 $\mu \mathrm{m}$ white polyester paint (including $5 \mu \mathrm{m}$ primer and $20 \mu \mathrm{m}$ top coat). The CCS panels were cut to the desired size with a guillotine from the top coat side downwards, and measured 30 $\mathrm{mm} \times 25 \mathrm{~mm}$. These coupons were placed horizontally in a flat-cell configuration at the bottom of the AC-SECM cell. The 3-electrode configuration, with the SECM tip as working electrode, a Pt-wire as counter, and a chlorinated silver wire as pseudo-reference electrode, was employed. The tips for the AC-SECM studies were glass insulated, disk-shaped Pt microelectrodes (25 $\mu \mathrm{m}$ disk diameter). All the experimental operations were performed with the substrate being unbiased, thus allowing the measurements to be carried out on the metal-coating system without any electrochemical perturbation.

Testing of coated samples to examine the effects on blistering susceptibility was carried out in sodium chloride solution of concentration $3 \%$ in weight for selected immersion times. Since AC-SECM operation must be performed in a low-conductivity electrolyte solution, all microelectrochemical measurements were performed in $1 \mathrm{mM}$ sodium sulphate solution, which was regarded to be inert towards blister initiation under the coating [6]. Electrolyteexchange was carefully performed in the SECM cell between subsequent treatment and testing steps. The electrolytes were prepared using twice-distilled water and analytical grade reagents (Sigma-Aldrich, Deisenhofen, Germany).

Frequency-dependent alternating-current scanning electrochemical microscopy (4D ACSECM) was employed for local visualization of blister initiation in coated metal samples upon immersion in chloride-containing electrolyte at room temperature. The sinusoidal voltage applied to the system was $100 \mathrm{mV}$ (peak to peak) in the frequency range from 351 to 55490 $\mathrm{Hz}$. The frequency values spaced logarithmically. The system AC response was analysed either in terms of the current magnitude $R$ and the phase shift $\theta$ or as real $(X)$ and imaginary $(Y)$ components of the $\mathrm{AC}$ current signal monitored. Approach curves $(R$ vs. $d)$ were 
recorded and used for tip positioning, even as a function of the $x$ - and $y$-tip position, whereas monitoring $R$ as a function of $x$ - and $y$-tip position allowed imaging. In this way, any unavoidable tilt of the sample could also be dynamically corrected by the instrument as to secure the tip was moved over the coated sample at a fixed distance. In this work, the operating tip-to-sample distance, was set at $12 \mu \mathrm{m}$, and images of area $700 \mu \mathrm{m} \times 700 \mu \mathrm{m}$ were obtained at a scan rate of $25 \mu \mathrm{m} \mathrm{s}^{-1}$.

Conventional EIS measurements were performed with Gamry equipment for the coated sample immersed in $3 \mathrm{wt} \% \mathrm{NaCl}$ aqueous solution for different time up to 24 hours. Impedance spectra were recorded at open corrosion potential in the frequency range 100 $\mathrm{kHz}$ to $10 \mathrm{mHz}$. The amplitude of the perturbing signal was $10 \mathrm{mV}_{\mathrm{pp}}$. The experiments were performed in duplicate, and no discrepancies were observed among them.

\section{3.- Results and discussion}

The highly resistive surface characteristics of the coil coated steel system upon exposure to an electrolytic environment could be demonstrated by recording conventional (global) impedance spectra after immersion in $3 \mathrm{wt} \% \mathrm{NaCl}$ aqueous solution. Figure 1 shows the Nyquist plots for two different immersion times up to 10 hours. The outstanding barrier effect conferred by the polymeric film for the protection against corrosion of the underlying metallic material can be concluded from the combined observation of only one time constant in the graphs, impedance magnitude values in excess of $1 \mathrm{M} \Omega \mathrm{cm}^{2}$ in the low frequency range, and an almost purely capacitive behaviour over the full frequency range. These features are common to all the spectra recorded for the duration of the current experiment.

Frequency-dependent alternating-current scanning electrochemical microscopy (4D ACSECM) is a technique especially suited for the investigation of such highly resistive surfaces. By applying an alternating potential to a microelectrode used as SECM-tip and recording the alternating current response, spatially-resolved information on the electrochemical behaviour of insulating samples becomes available if the system is operated in sufficiently diluted electrolytes. Low conductivity of the electrolytic phase is required if the current should flow to the sample directly below the tip and the technique be sensitive to the topography of the surface. This effect can be explained by using the phenomenological equivalent circuit depicted in figure 2, which is a simple description of the different contributions affecting the overall response of the system. They can be reduced to a resistor in the case of the solution resistance $R_{\text {sol }}$, and to a parallel $R C$ combination for every reacting interface. In this way, $R_{T}$ 
and $C_{T}$ represent the resistance and the capacitance of the tip; $R_{S}$ and $C_{S}$, the resistance and capacitance of the coated sample; and $R_{S^{\prime}}$ and $C_{S^{\prime}}$, the local resistance and capacitance of the coated sample at the specific area covered by the AC-SECM tip, respectively. Finally, $R_{\text {sol }}$ has been depicted as a variable resistor because it changes with the absolute $z$-distance between the tip and the sample surface, which is the origin of the near-field response observed in the system.

A word of caution must be taken in relation that we do not claim that a single time constant (expressed in terms of an $R C$ combination) corresponds to each interface. They are actually "experimental $R$ and $C$ " values, and they would have to be modelled to the relevant physicochemical process to match their observed frequency dependence when used as local impedance measurements. Indeed, it must be realized that not necessarily the $R_{\mathrm{s}}$ values as described in the previous paragraph can directly be replaced by a faradaic impedance, as this will not be the case in many actual systems.

Thus, depending on the applied frequency and the electrochemical characteristics of the surface, the current will preferentially flow through one of the pathways depicted in figure 2 depending on their corresponding impedances. In this case, the capacitive impedance dominates over the solution impedance while it varies inversely with the frequency of the $A C$ excitation. A negative feedback type effect is to be expected for this insulating system when the tip slowly approaches the surface of the coated sample whereas simultaneously recording the measured AC current at the microelectrode vs. $z$ displacement. Indeed, a negative feedback trend is found when recording the approach curve towards the intact coated sample immersed in $1 \mathrm{mM}$ sodium sulphate as the test solution, as it is shown in figure 3. In this way, the true distance between the tip and the substrate could be determined from the height of closest approach at which the microelectrode displacement was stopped.

Once the true distance between the tip and the substrate was determined, the tip was withdrawn from the sample surface to a distance equivalent to half the active area of the microelectrode, namely $12 \mu \mathrm{m}$. Then, surface imaging was performed by moving the tip parallel to the surface. In this case, a frequency spectrum was applied at each point of the scanning grid and after the scan was completed, the images were composed for each frequency. Selected images of the sample using the current amplitude for an intact coated sample shortly after immersion in $1 \mathrm{mM} \mathrm{Na}_{2} \mathrm{SO}_{4}$ solution are shown in figure 4A. Clearly, a topographically flat surface is observed at all frequencies, even at the higher frequencies used which provide the highest contrast [22]. All the 3D maps shown were recorded by shifting the microelectrode tip from left to right (i.e., they were built as a composition of $x$ 
displacement lines, which were stepwise shifted in the $y$ direction to the back of the images). The apparent tilt effect originates from the processes that occur inside the electrochemical cell during an experiment, namely the different extent of water uptake by the coating as the time elapses. That is, the permeation of water into the polymeric matrix results in an increase of its volume, and the surface thus progressing into the solution phase [23]. In the SECM system, this process originates the surface of the substrate effectively approaching the tip when moved at the same height continuously over the substrate. Thus, the line scans at the back of the image, effectively measured at later times, were thus recorded above a protruded surface compared to the beginning of the imaging process. This happened though dynamic tilt correction was performed on the system, because it was based on the approach curves measured at the beginning of the experiment and they could not compensate the dynamic process of water uptake by the coating.

After image collection was completed, the tip was removed from the electrolyte by using the $z$-positioning control. Next, electrolyte exchange was performed to investigate the effect of chloride ions on the coated system. In this way, the coated sample could be exposed to 3 wt.\% $\mathrm{NaCl}$ solution for selected times. Visualization of the surface by AC-SECM could be performed after a new electrolyte exchange, that is, when the sample was immersed in $1 \mathrm{mM}$ $\mathrm{Na}_{2} \mathrm{SO}_{4}$ solution again. The tip was then introduced in the electrolytic phase, and the corresponding approach curves were recorded to accurately establish the required tipsample distance for imaging. The process could be repeated as many times as necessary as to adequately follow the changes occurring in the topography of the coated metal due to its exposure to a chloride-containing electrolyte.

Figures $4 \mathrm{~B}$ and $4 \mathrm{C}$ show the actual images of the sample after 5 and 72 hours exposure to 3 $w t . \% \mathrm{NaCl}$ solution, respectively. As the sample surface has insulating characteristics towards the flow of current, any variations observed in the AC-SECM images recorded after different exposures to the chloride-containing test environment must be due to modifications of the surface topography of the substrate. A trend towards the development of increased surface roughening with the elapse of time is deduced from the comparison of the images passing from column $(A)$ to column $(C)$ in Figure 4 , this feature being more noticeable for the higher $A C$ frequencies used in this work. Furthermore, the extent of this effect is observed to be progressing with the elapse of time, i.e., it is rather small in the sample exposed to $\mathrm{NaCl}$ solution for 5 hours, and it is very intense after 72 hours. Even some early blistering of the coated sample can be found for the longest exposure. 
It must be noted at this point that AC-SECM images have always been recorded at a constant height of $12 \mu \mathrm{m}$ above the coated sample, instead of maintaining the tip at the same absolute position in the Z-axis. This condition would effectively result in the tip being kept at a constant distance from the underlying metal surface instead to the surface of the coating. In that case, the net effect of water uptake resulting in the coated surface effectively approaching the tip would result in the images being obtained with shorter tip-sample distances as time elapses, thus allowing the surface topography to be recorded with increasing lateral resolution. If that would have been the case, the observation of more details in the images would be just an artefact resulting from the varying tip-sample distance along the experiment. On the contrary, we have chosen to operate at a constant tip-sample distance by repeating the sequence of tip approach curves recorded just before starting to record the AC-SECM map, effectively allowing for the constant height of $12 \mu \mathrm{m}$ to be restored and the dynamic tilt correction to be applied.

This finding has important consequences towards the mechanistic description of coating degradation through blistering, which is usually considered to be initiated at significantly longer exposures than the times involved in water uptake, always involving the prior formation of continuous ionic pathways through pores in the organic film until the underlying metal is effectively exposed to the electrolytic phase at the bottom of those pores. But the evidence given here that chloride ions have a specific effect consistent with their transport through the still "intact" coating, either accumulating at locations within the polymer matrix, or most probably accumulating at the underlying metal/polymer interface, introduces a new scenario. The metal/polymer interface is known to contain a thin layer of water, and the arrival of chloride ions to this interface effectively originates a rather concentrated saline solution that would impose a growing osmotic pressure through the coating as the solution accumulates at preferential sites of possibly lower adhesion. These growing osmotic pressure would thus be the leading force towards blister formation in the system.

\section{4.- Conclusions}

Direct evidence has been found of surface roughening consistent with the early stages of blistering under an organic coating applied on a metal as promoted by the action of chloride ions present in the environment. This effect is found to occur almost simultaneous to the uptake of water by the electrolyte, certainly well before ionic pathways through the coating could have been developed, as confirmed by the invariability of coating resistance within this time scale as measured by electrochemical impedance spectroscopy. 


\section{Acknowledgements:}

The authors are grateful to the German Academic Exchange Service (DAAD, Bonn) and to the Spanish Ministry of Science and Innovation (MICINN, Madrid, Acción Integrada No. HA2006-0077) for the grant of a Collaborative Research Programme between Germany and Spain. M.P. and W.S. are grateful for financial support by the DFG in the framework of the SFB459 (A5) and the project "Elucidation of corrosion phenomena with high lateral resolution using scanning probe techniques" (SCHU 929/9-1).

\section{References:}

1. L.L. Shreir, R.A. Jarman, G.T. Burstein (Eds.), Corrosion, vol. 2, third ed.; ButtherworthHeinemann, Oxford, 1994, Chapter 14.

2. J.H.W. de Wit, in: P. Marcus, J. Oudar (Eds.), Corrosion Mechanisms in Theory and Practice; Marcel Dekker, New York, 1995, p. 581.

3. G. Grundmeier, A. Simões, in: A.J. Bard, M. Stratmann (Eds.), Encyclopedia of Electrochemistry, vol. 4; Wiley-VCH, Weinheim, 2003, p. 499.

4. A. Forsgren, Corrosion Control Through Organic Coatings; CRC Press, Boca Raton, 2006.

5. J.E.O. Mayne, in: L.L. Shreir, R.A. Jarman, G.T. Burstein (Eds.), Corrosion, vol. 2, third ed.; Butterworths-Heinemann, Oxford, 1994, p. 14:22.

6. R.M. Souto, Y. González-García, S. González, G.T. Burstein, Corrosion Science, 46, 2621-2628 (2004).

7. R.M. Souto, Y. González-García, S. González, Corrosion Science, 50, 1637-1643 (2008).

8. R.M. Souto, Y. González-García, S. González, G.T. Burstein, Electroanalysis, 21, 25692574 (2009).

9. R.M. Souto, Y. González-García, J. Izquierdo, S. González, Corrosion Science, 52, 748753 (2010).

10. R.M. Souto, L. Fernández-Mérida, S. González, D.J. Scantlebury, Corrosion Science, 48, 1182-1192 (2006).

11. Y. González-García, S. González, R.M. Souto, Corrosion Science, 49, 3514-3526 (2007).

12. A.J. Bard, F.-R. Fan, M. Mirkin, in: I. Rubinstein (Ed.), Physical Electrochemistry: Principles, Methods, and Applications; Marcel Dekker, New York, 1995, p. 209.

13. A.J. Bard, M.V. Mirkin (Eds.), Scanning Electrochemical Microscopy. Marcel Dekker, New York, 2001. 
14. R.M. Souto, Y. González-García, S. González, Progress in Organic Coatings, 65, 435439 (2009).

15. B. Ballesteros Katemann, A. Schulte, E.J. Calvo, M. Koudelka-Hep, W. Schuhmann, Electrochemistry Communications, 4, 134-138 (2002).

16. M. Etienne, A. Schulte, W. Schuhmann, Electrochemistry Communications, 6, 288-293 (2004).

17. A.S. Baranski, P.M. Diakowski, Journal of Solid State Electrochemistry, 8, 683-692 (2004).

18. K. Eckhard, W. Schuhmann, Analyst, 133, 1486-1497 (2008).

19. K. Eckhard, T. Erichsen, M. Stratmann, W. Schuhmann, Chemistry European Journal, 14, 3968-3976 (2008).

20. M. Pähler, J.J. Santana, W. Schuhmann, R.M. Souto, Chemistry European Journal, 17, 905-911 (2011).

21. P.M. Diakowski, A.S. Baranski, Electrochimica Acta, 52, 854-862 (2006).

22. K. Eckhard, W. Schuhmann, M. Maciejewska, Electrochimica Acta, 54, 2125-2130 (2009).

23. Y. González-García, J.J. Santana, J. González-Guzmán, J. Izquierdo, S. González, R.M. Souto, Progress in Organic Coatings, 69, 110-117 (2010).
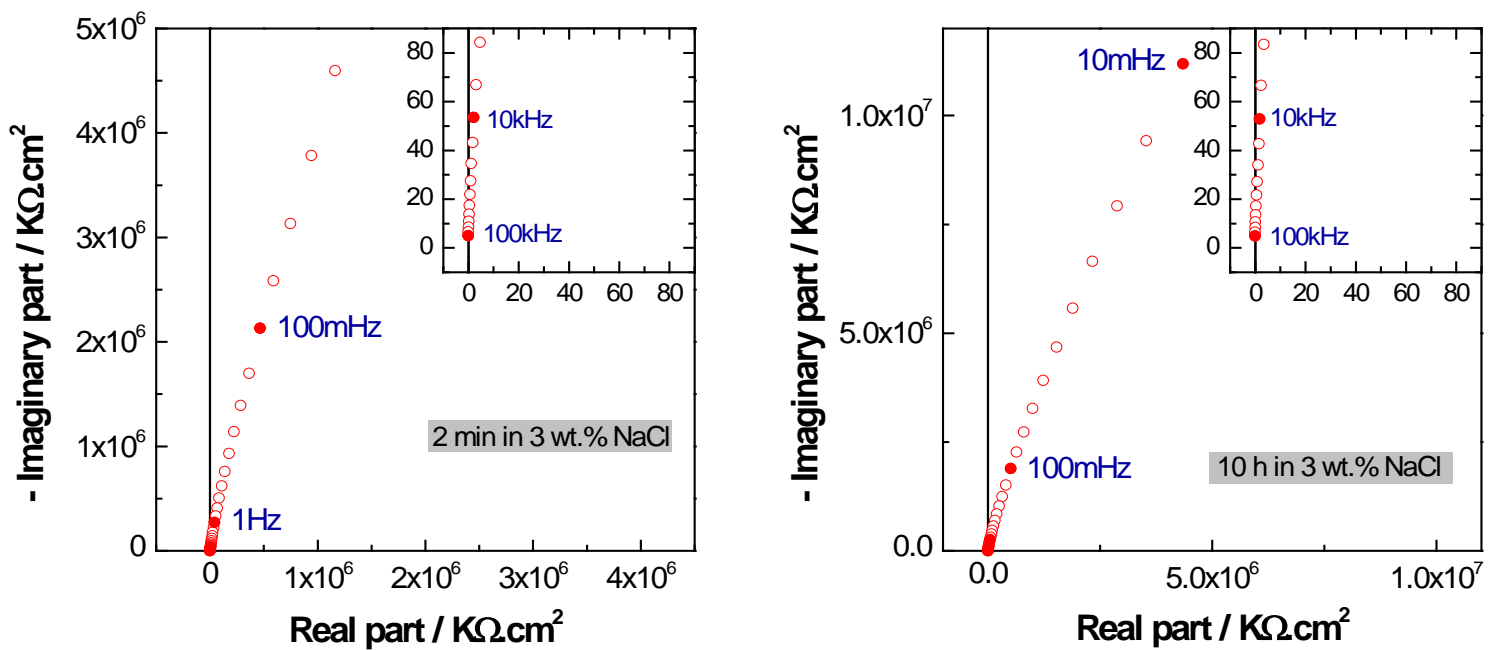

Figure 1: EIS spectra of a painted sample after immersion in $3 \mathrm{wt} . \% \mathrm{NaCl}$ aqueous solution for two different times, namely 2 min and 10 hours. 


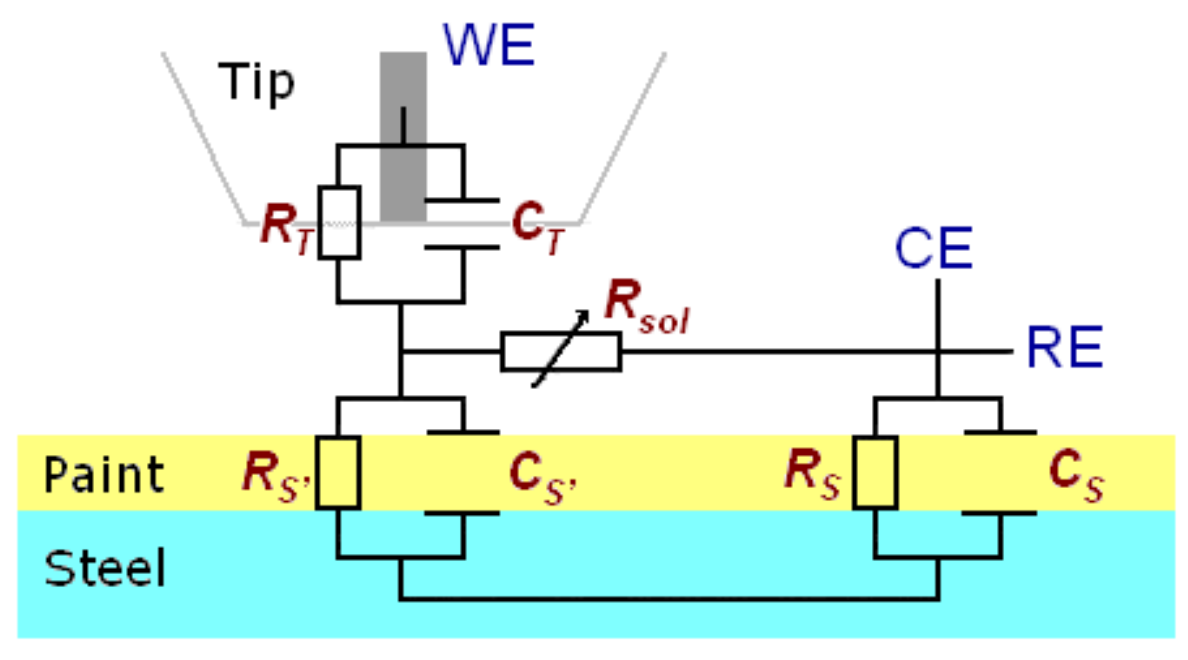

Figure 2: Simplified equivalent circuit representing the impedance behaviour for a coated metal exposed to an aqueous electrolyte in an AC-SECM arrangement [19]. $R_{\mathrm{T}}$ and $C_{\mathrm{T}}$ are resistance and capacitance of the tip; $R_{\mathrm{sol}}$ is the solution resistance; $R_{\mathrm{S}}$ and $C_{\mathrm{S}}$ are the resistance and capacitance of the coated sample; $R_{S^{\prime}}$ and $C_{S^{\prime}}$ are the local resistance and capacitance of the coated sample at the specific area covered by the AC-SECM tip.

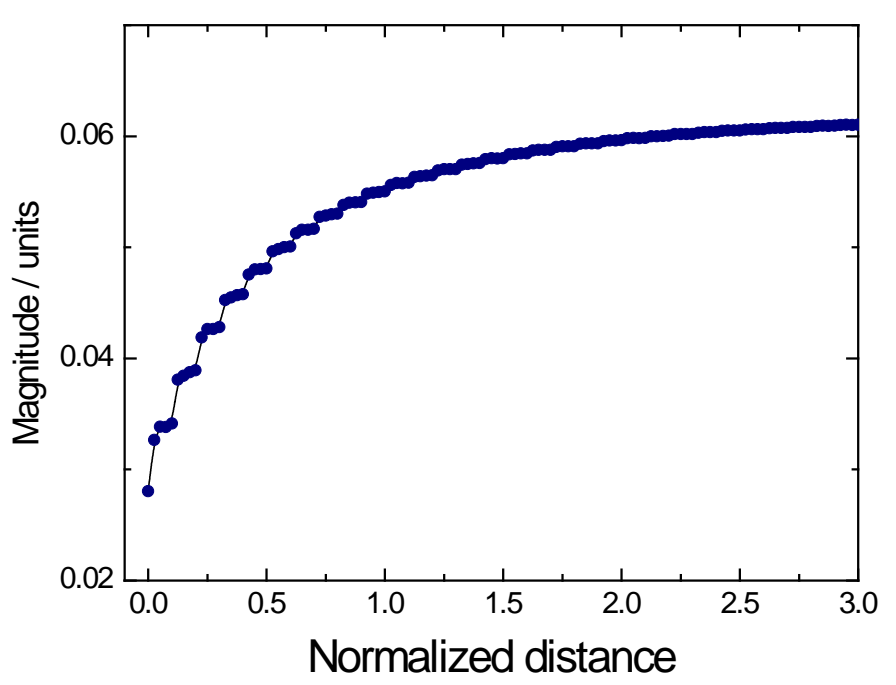

Figure 3: Normalized approach curve towards a painted sample immersed in a $1 \mathrm{mM}$ $\mathrm{Na}_{2} \mathrm{SO}_{4}$ solution with a $25 \mu \mathrm{m}$ Pt electrode. The excitation signal amplitude was $100 \mathrm{mV}_{\mathrm{pp}}$ and the frequency $4413 \mathrm{~Hz}$. The sample was approached in the test electrolyte prior to its exposure in the $3 \mathrm{wt} . \% \mathrm{NaCl}$ aqueous solution. 
A)

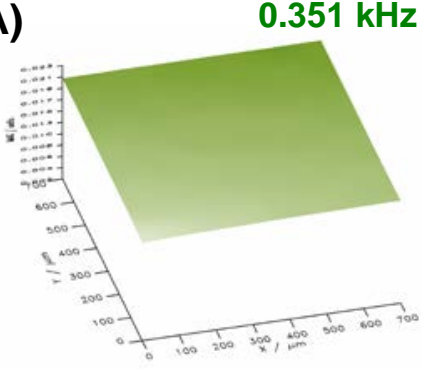

$1.245 \mathrm{kHz}$

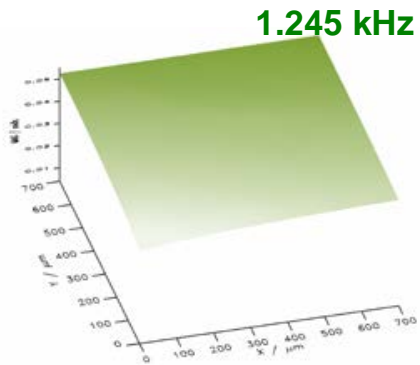

$4.413 \mathrm{kHz}$

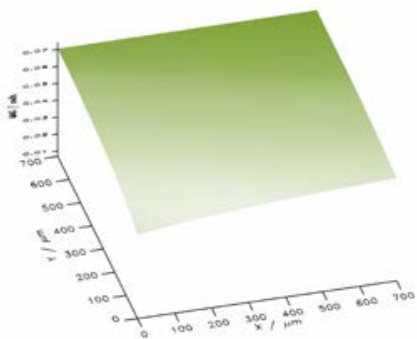

$8.310 \mathrm{kHz}$

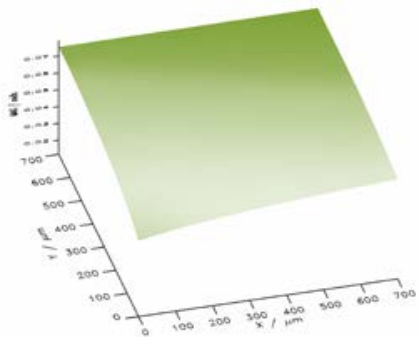

$24.469 \mathrm{kHz}$

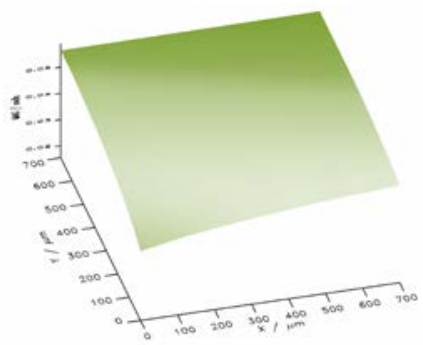

B)

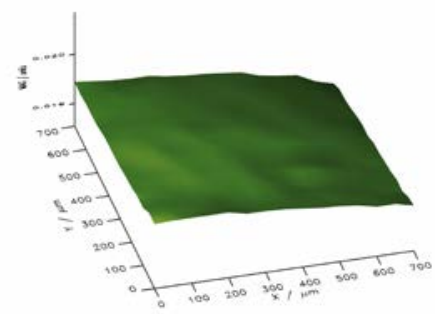

$1.245 \mathrm{kHz}$

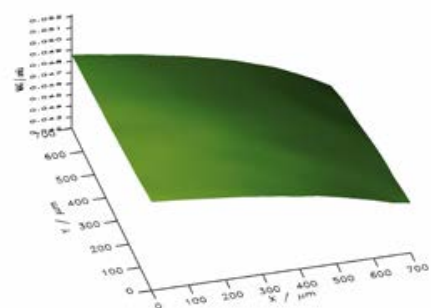

$4.413 \mathrm{kHz}$

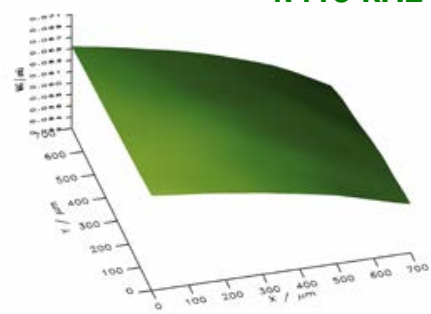

$8.310 \mathrm{kHz}$

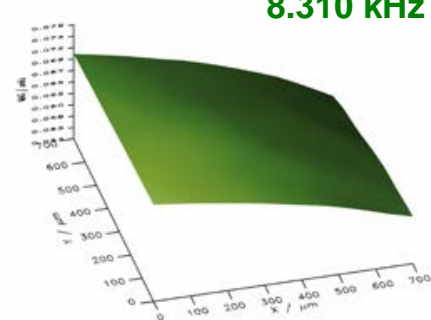

$24.469 \mathrm{kHz}$

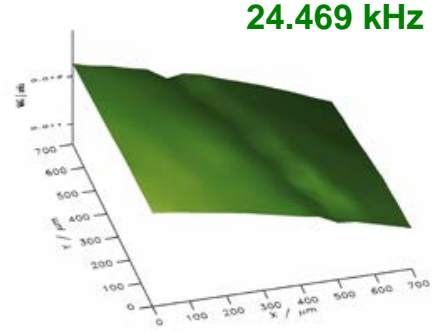

C)

$0.351 \mathrm{kHz}$
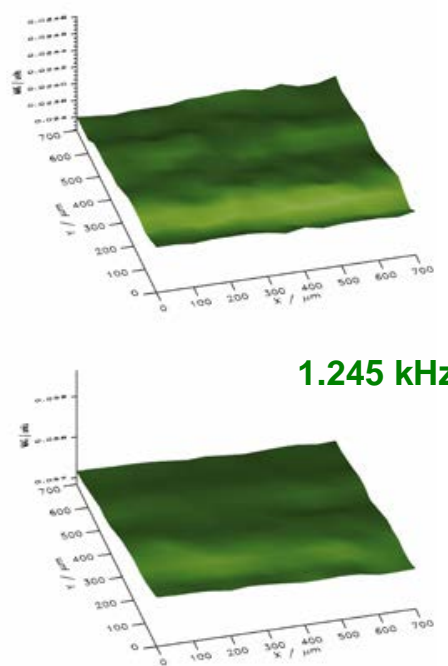

$4.413 \mathrm{kHz}$

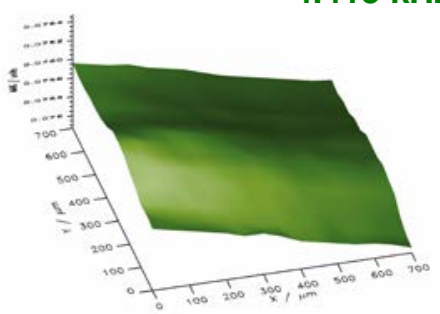

$8.310 \mathrm{kHz}$

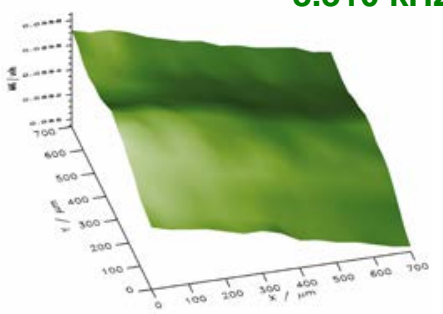

$24.469 \mathrm{kHz}$

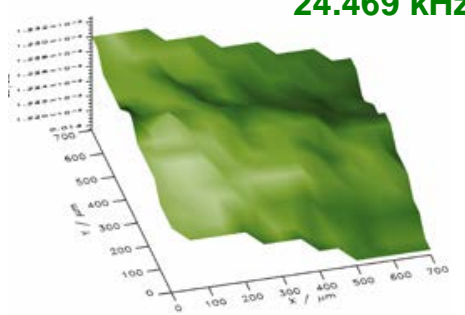

Figure 4: Images generated by AC-SECM measurements on a painted sample both prior $(A)$ and after exposure $(B, C)$ to $3 \mathrm{wt} . \% \mathrm{NaCl}$ solution for selected times. The images were taken after electrolyte exchange during immersion in $1 \mathrm{mM} \mathrm{Na}_{2} \mathrm{SO}_{4}$ solution, and they represent $700 \mu \mathrm{m} \times 700 \mu \mathrm{m}$ in $x$ and $y$ directions. Images in column (A) were taken immediately before initiating the exposure of the specimen to the chloride-containing electrolyte, whereas those in columns (B) and (C) were taken after the specimen was exposed during 5 and 72 hours, respectively. Tip diameter $(2 r)=25 \mu \mathrm{m}$; Tip-substrate distance $(d)=13 \mu \mathrm{m}$. Alternating current signal applied to the tip: amplitude $\left(V_{p p}\right)=100 \mathrm{mV}$, frequency range $(f): 0.351 \rightarrow$ $24.469 \mathrm{kHz}$, in 9 logarithmic increments. 